\title{
Appendiceal Diverticulitis in a Young Female Diagnosed on Pathology after Laparoscopic Appendectomy for Acute Appendicitis
}

\author{
Oluwatobi O. Onafowokan $\mathbb{D}^{1,2}$ Aboubakr Khairat $\mathbb{D}^{1},{ }^{1,3}$ and Hugo J. R. Bonatti $\mathbb{D}^{1,3}$ \\ ${ }^{1}$ University of Maryland Community Medical Group, Easton, MD, USA \\ ${ }^{2}$ Royal Lancaster Infirmary, Lancaster, UK \\ ${ }^{3}$ Meritus Surgical Specialists, Hagerstown, MD, USA
}

Correspondence should be addressed to Hugo J. R. Bonatti; hugo.bonatti@dr.com

Received 1 May 2020; Revised 30 January 2021; Accepted 1 March 2021; Published 8 March 2021

Academic Editor: John Kortbeek

Copyright (C) 2021 Oluwatobi O. Onafowokan et al. This is an open access article distributed under the Creative Commons Attribution License, which permits unrestricted use, distribution, and reproduction in any medium, provided the original work is properly cited.

\begin{abstract}
Background. Appendiceal diverticulitis is a rare cause of inflammation of the appendix, which may mimic acute appendicitis. Its diagnosis is often delayed, and its occurrence carries an increased risk of significant complications, such as perforation. Case Presentation. A 23-year-old woman presented with sudden onset, severe, right lower quadrant abdominal pain and nausea. Her WBC was elevated, and abdominal CT showed findings indicative of acute appendicitis with a $13 \mathrm{~mm}$ fluid-filled appendix and local stranding. During laparoscopic appendectomy, significant inflammation was found around the appendix with some mucous material around the tip. The appendix base was not involved, and an endoloop was used to secure the stump. No other intraabdominal abnormalities were observed. The patient recovered uneventfully. Pathology showed no classic appendicitis but appendiceal diverticulitis with signs of perforation. Discussion. Appendiceal diverticulitis is a rare condition which cannot be distinguished from acute appendicits clinically and on imaging. Diagnosis may be established based on pathology such as in our case. Appendectomy is indicated in appendiceal diverticulitis, and an appendix diverticulum is incidentally found during surgery or other investigations. This is due to the increased risk of perforation and the reported development of malignant tumors, including the appendix carcinoid.
\end{abstract}

\section{Introduction}

Appendiceal diverticulosis is an uncommon pathology, first described in 1893 [1-3]. Congenital appendiceal diverticulosis is a true diverticulum, with a rare incidence of $0.014 \%$ [4]. Acquired appendiceal diverticulosis is a false diverticulum on the mesenteric border of the appendix, with a relatively more common incidence of $1.9 \%$. Pathogenesis of acquired appendiceal diverticulosis is not completely understood, but it may be associated with diverticulosis of other colonic segments, and various pathologies of the appendix $[3,5]$.

Appendiceal diverticulitis occurs due to the inflammation of an appendiceal diverticulum [2,6]. It is a rare cause of inflammation of the appendix, which may mimic acute appendicitis [5, 7]. Its diagnosis may be delayed, and its occurrence carries an increased risk of significant complications, including perforation [8] and a higher risk of mortality $[2,4,9]$. Progression of diverticulosis to diverticulitis may occur following a partial or complete obstruction of the appendix lumen. This obstruction may be due to inflammation, mucosal swelling, fecaliths, torsion, or fibrous strictures [2].

Appendectomy is recommended when appendiceal diverticulosis is incidentally discovered on imaging or during surgery as up to $66 \%$ of patients will progress to an acute inflammation [2]. Other authors have considered prophylactic appendectomy less beneficial [10]. The predominant scenario in the majority of publications involves patients diagnosed with acute appendicitis and undergoing appendectomy, but with final pathology showing appendiceal diverticulitis and not appendicitis. It should be noted that 


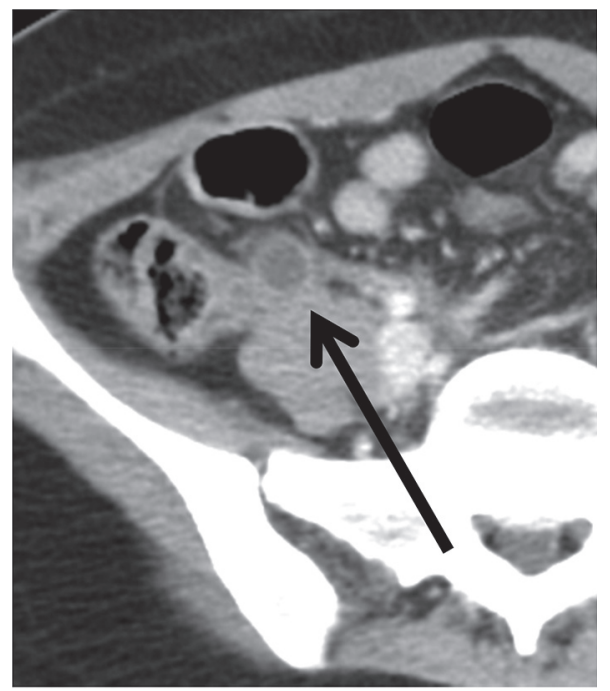

(a)

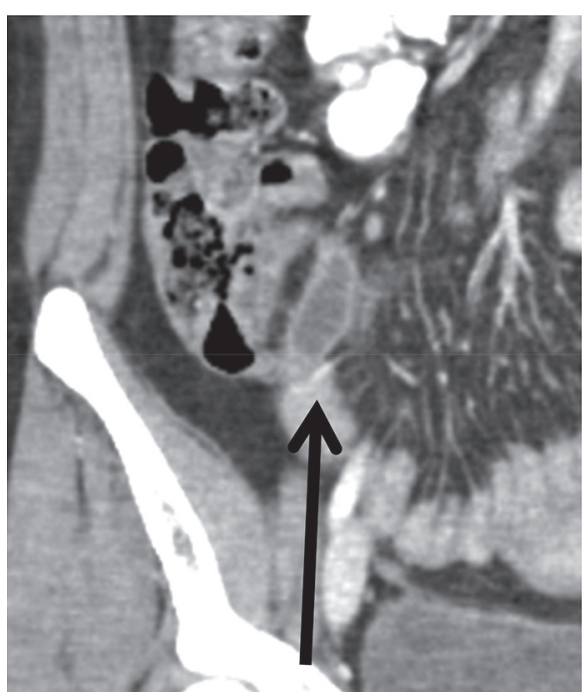

(b)

FIGURE 1: CT scan (transverse and coronal cut). Thickened fluid-filled appendix with localized fat stranding; an appendiceal diverticulum cannot be seen.

both inflammatory processes may occur simultaneously according to the classification proposed by Lipton et al. [8].

We report the clinical course of a young female who presented with an episode of right lower quadrant pain with a clinical and computed tomography (CT) scan diagnosis of acute appendicitis. She underwent laparoscopic appendectomy and was found to have appendiceal diverticulitis on final pathology.

\section{Case Presentation}

A 23-year-old healthy woman presented to the emergency room (ER) with acute onset, severe right lower quadrant (RLQ) pain and nausea. On examination, she was alert and oriented, her abdomen was soft, but she had significant tenderness in the RLQ and a positive Murphy's sign. White blood count was normal, and abdominal computed tomography (CT) scan showed a fluid-filled and dilated appendix with thickened walls and localized inflammatory changes in the RLQ indicative of acute appendicitis (Figure 1).

She was started on antibiotics in the ER (ertapenem $1 \mathrm{~g}$ ) and consented for laparoscopic appendectomy. Laparoscopy was done with $5 \mathrm{~mm}$ left (L) UQ and umbilical trocars and a suprapubic Teleflex MiniLap Alligator Grasper [11]. The appendix was dilated and acutely inflamed with mucosal secretions on the tip of the appendix (Figure 2). The vascular pedicle and appendix base were secured with endoloops.

The patient was discharged the next morning and had an uneventful recovery. Final pathology revealed a diverticulum in the tip of the appendix, with active inflammation (Figure 3).

\section{Discussion}

Acute appendicitis due to appendiceal diverticulitis is a rare condition, with an incidence of $0.004-2.1 \%$ [7]. Risk factors may include age $>30$ years old, male gender, and comorbidities

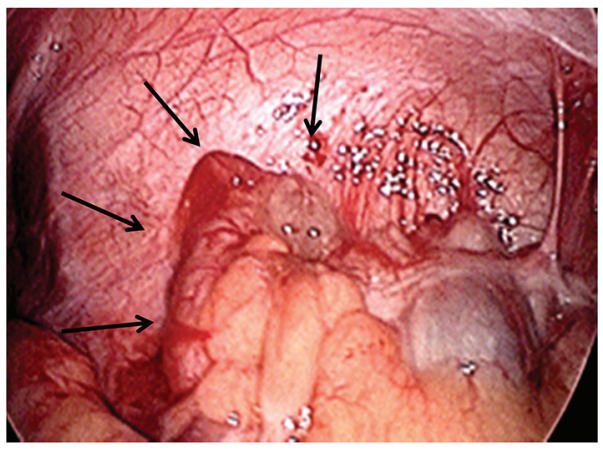

FIGURE 2: Intraoperative findings: periappendicitis and acute inflammation of the appendix with mucinous secretions.

such as cystic fibrosis [7]. Diagnosis is most frequently incidental but may be made my ultrasound or CT scan in selected cases [9]. Appendiceal diverticulitis has been shown to be more than four times as likely to perforate compared with acute appendicitis, increasing mortality 30-fold compared with simple appendicitis $[2,8]$. Therefore, correct diagnosis and urgent management with antibiotics and appendectomy are currently favoured with laparoscopy being the preferred approach $[12,13]$. In contrast to diverticulitis of the colon or small bowel, no large series demonstrating successful nonoperative management of appendiceal diverticulitis are available, and this is most likely due to the fact that appendicitis and appendiceal diverticulitis are difficult to distinguish on imaging [14]. Radiographic diagnosis of appendiceal diverticulosis/diverticulitis is difficult, but CT scan may identify an appendiceal diverticulum with the pericaecal fat showing increased density.

It should be recognized that diverticula of the appendix are also associated with an increased risk of appendiceal neoplasms including carcinoid tumors, adenocarcinoma, and mucinous adenomas (pseudomyxoma peritonei) [4]; strengthening the argument for appendectomy $[13,15]$. 


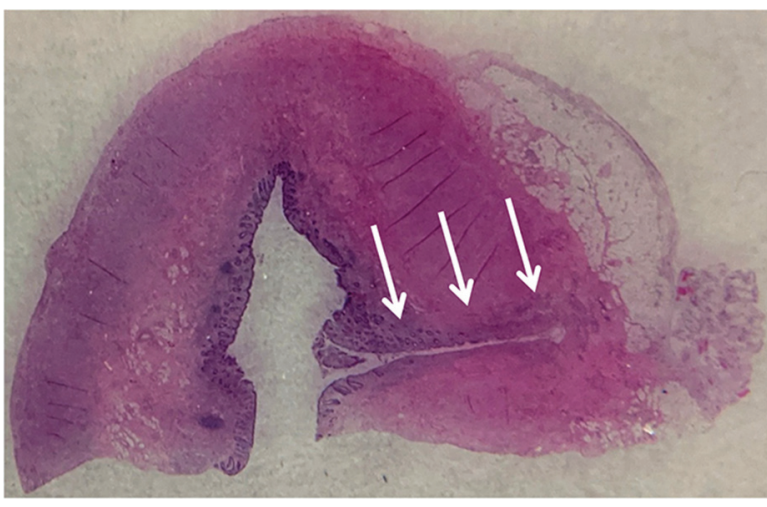

(a)

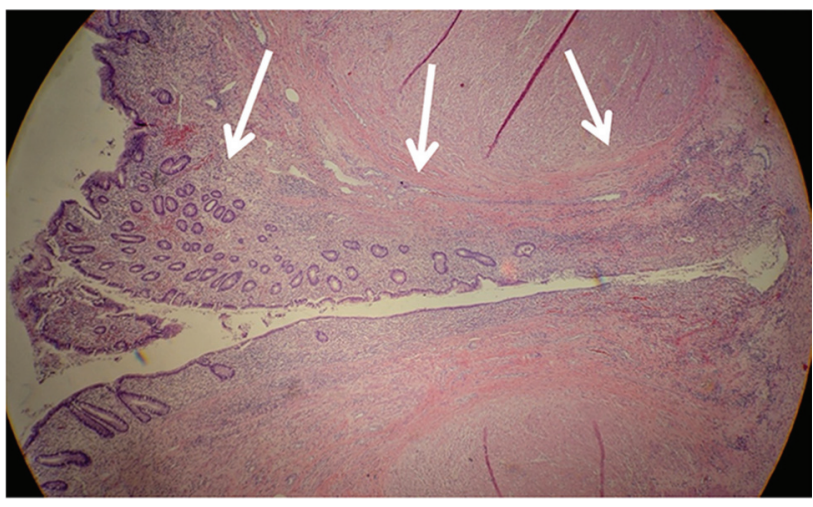

(b)

Figure 3: Pathology findings: section of the appendix tip (a) and close up (b) of the diverticulum (arrows) showing acute inflammation.

To summarise, acute appendicitis due to appendiceal diverticulitis is an uncommon condition, which is often diagnosed on pathology. Appendiceal diverticulitis may be associated with significant complications. Currently, appendectomy is favoured for acute appendiceal diverticulitis and incidentally discovered appendiceal diverticulosis, with laparoscopy being the preferred approach.

\section{Disclosure}

Parts of this article were presented as a poster at the 60th Annual Meeting of the Austrian Society of Surgery in June 2019 in Innsbruck, Austria (https://link.springer.com/ article/10.1007/s10353-019-0600-2).

\section{Conflicts of Interest}

The authors declare that they have no conflicts of interest.

\section{References}

[1] T. N. Kelynack, "A contribution to the pathology of the vermiform appendix," Glasgow Medical Journal, vol. 41, no. 1, pp. 67-68, 1894.

[2] B. J. Phillips and C. W. Perry, "Appendiceal diverticulitis," Mayo Clinic Proceedings, vol. 74, no. 9, pp. 890-892, 1999.

[3] D. C. Collins, "A study of 50,000 specimens of the human vermiform appendix," Surgery, Gynecology \& Obstetrics, vol. 101, no. 4, pp. 437-445, 1955.

[4] M. P. Dupre, I. Jadavji, E. Matshes, and S. J. Urbanski, "Diverticular disease of the vermiform appendix: a diagnostic clue to underlying appendiceal neoplasm," Human Pathology, vol. 39, no. 12, pp. 1823-1826, 2008.

[5] I. Yamana, S. Kawamoto, K. Inada, S. Nagao, T. Yoshida, and Y. Yamashita, "Clinical characteristics of 12 cases of appendiceal diverticulitis: a comparison with 378 cases of acute appendicitis," Surgery Today, vol. 42, no. 4, pp. 363-367, 2012.

[6] D. S. Heffernan, N. Saqib, and M. Terry, "A case of appendiceal diverticulitis, and a review of the literature," Irish Journal of Medical Science, vol. 178, no. 4, pp. 519-521, 2009.

[7] B. Abdullgaffar, "Diverticulosis and diverticulitis of the appendix," International Journal of Surgical Pathology, vol. 17, no. 3, pp. 231-237, 2009.
[8] S. Lipton, J. Estrin, and I. Glasser, "Diverticular disease of the appendix," Surgery, Gynecology \& Obstetrics, vol. 168, no. 1, pp. 13-16, 1989.

[9] M. L. Altieri, G. N. Piozzi, P. Salvatori, M. Mirra, G. Piccolo, and N. Olivari, "Appendiceal diverticulitis, a rare relevant pathology: presentation of a case report and review of the literature," International Journal of Surgery Case Reports, vol. 33, pp. 31-34, 2017.

[10] H. Kabiri, L. E. Clarke, and C. D. Tzarnas, "Appendiceal diverticulitis," The American Surgeon, vol. 72, no. 3, pp. 221-223, 2006.

[11] H. J. R. Bonatti, "Development of a two port laparoscopic appendectomy technique at a rural hospital," Minimally Invasive Surgery, vol. 2019, no. 8, 7 pages, Article ID 9761968, 2019.

[12] M. Z. Albeeshi, A. A. Alwanyan, A. A. Salim, and I. T. Albabtain, "Appendiceal diverticulitis presenting as acute appendicitis diagnosed postoperatively," Journal of Surgical Case Reports, vol. 2019, no. 12, p. rjz332, 2019.

[13] J. L. Ng, S. L. Wong, and R. Mathew, "Appendiceal diverticulosis: a harbinger of underlying primary appendiceal adenocarcinoma?" Journal of Gastrointestinal Oncology, vol. 9, no. 2, pp. E1-E5, 2018.

[14] A. H. Yardimci, C. T. Bektas, E. Pasaoglu et al., "Retrospective study of 24 cases of acute appendiceal diverticulitis: CT findings and pathological correlations," Japanese Journal of Radiology, vol. 35, no. 5, pp. 225-232, 2017.

[15] S. A. Käser, N. Willi, and C. A. Maurer, "Prevalence and clinical implications of diverticulosis of the vermiform appendix," Journal of International Medical Research, vol. 41, no. 4, pp. 1350-1356, 2013. 